\title{
Lymphocytes play the music but the macrophage calls the tune
}

\author{
Werner Solbach, Heidrun Moll and \\ Martin Röllinghoff
}

Researchers interested in immunological aspects of bacterial, fungal, protozoan and helminthic infection are too often kept apart by artificial subvacuoles that exclude host membrane glycoproteins. Such vacuoles fail to fuse with acidifying lysosomes and endosomes thus protecting the parasites from lethal host responses. However, if the tachyzoites are coated with antibody, they are bound by macrophage $\mathrm{Fc}$ receptors $(\mathrm{FCR})$; the blocks in fusion and acidification of the vacuoles are overcome and the microbes are killed by triggering the macrophage's respiratory burst. The mechanism of this FcRmediated reversal of the block in fusion and acidification is not yet clear but studies using respiratoryburst-negative cells transfected with various FcR mutants have shown that distinct domains of the cytoplasmic tail of the FcR direct endocytosis and phagocytosis.

Leishmania adopt a different entry and survival strategy. They passively attach to various receptors, including complement receptors, on the host cell surface by means of lipophosphoglycan and gp63 and are taken up by conventional phagocytosis. Leishmania-containing phagosomes fuse effectively with lysosomes, as shown by the presence of lysosomal enzymes within the parasitophorous vacuole (D. Russell; St Louis) and kinetic studies have revealed that a constant supply of lysosomal enzymes is likely to be released into these vacuoles. The ject boundaries. These barriers were temporarily breached by a recent workshop* in which the complex interplay between microbes and their mammalian hosts were examined from a global viewpoint. The role of T-cell subsets and their products came under close scrutiny but the most forceful image was that of the macrophage. As host for infective agents, as modulator of specific immune activity and as ultimate mediator of the host response, the macrophage plays a virtuoso's role in the host-parasite drama.

for antigen presentation: the metabolic inhibitor Brefeldin-A abrogated the presentation of parasite antigens to MHC class-II-restricted $T$ cells. Brefeldin-A blocks the transport of newly synthesized proteins from the endoplasmic reticulum to the Golgi apparatus, suggesting that only nascent $\mathrm{MHC}$ molecules are used by APCs.

The macrophage as an APC plays a key role in determining whether or not a cellular immune response promotes protection of the host or survival of the infectious agent. One important factor is the level of expression of MHC molecules; as an illustration of this, H-D. Volk magnitude of the respiratory burst is decreased when triggered by complement-coated parasites, so that the mechanism of entry may contribute in more than one way to the survival of the parasite.

Macrophages as antigen-presenting cells (APCs)

Two major pathways have evolved for the presentation of antigen to $T$ cells. Intracellular antigens are mainly processed in the cytosol and are presented in the context of MHC class I molecules, whereas exogenous antigens are generally processed by the endosomal pathway and associate with $\mathrm{MHC}$ class II molecules. P. Kaye (London), working with Leishmania donovani, showed that phagolysosomes of infected macrophages contain parasites in various stages of degradation. Although MHC class II molecules could be seen within the phagolysosomes, they are not used

*The Fourth International Erlangen Workshop on Molecular Aspects of Immunological Host-Parasite Interactions was held at Erlangen, FRG on 27-29 September 1990. It was organized by $M$. Röllinghoff, W. Solbach and J. Kalden. (C) 1991, Elsevier Scicnce Publishers Let, UK. 0167-4919/91/S02.00
(Berlin) showed data demonstrating that in patients with septicemia there is a close association between reduction in monocyte HLA-DR expression and fatal outcome.

MHC antigen expression is modulated by T-cell-derived cytokines but can also be profoundly influenced by the natural resistance gene $B c g$ (which is probably identical to Ity and $L s h) . B c g$ is located on mouse chromosome 1 and, presumably, on human chromosome $2 \mathrm{q}$, within a set of genes coding for cytoskeletal proteins (E. Skamene, Montreal). In its two allelic forms $B c g$ confers resistance and susceptibility to infection with Salmonella typhimurium, Leishmania donovani, mycobacteria and other macrophagic parasites in mice and preliminary studies in humans suggest a similar dichotomy. The $B c g$ gene product, in addition to controlling $\mathrm{MHC}$ class II antigen expression, affects $5^{\prime} \mathrm{nu}$ cleotidase activation, the respiratory burst and expression of the ligand for AcM.1, an antibody that recognizes activated macrophages.

MHC molecules direct the immune system towards responses mediated largely by either $\mathrm{CD}^{+}$or $\mathrm{CD} 8^{+} \mathrm{T}$ cells and this may be partly 
controlled by the site of intersection of MHC molecules and peptide. Thus infected macrophages may present a distinct set of peptides, and induce a response of different emphasis to that induced by uninfected macrophages that acquire locallyavailable peptides by endocytosis. The suggestion by J. Louis (Lausanne) that live and dead parasites induce different $\mathrm{T}$-cell subsets is in agreement with this possibility. The divergent responses induced may be synergistic: in cutaneous leishmaniasis, resistance to disease is induced by $\mathrm{CD} 4^{+}$cells without the requirement for $\mathrm{CD} 8^{+}$cells. How ever, $\mathrm{CD}^{+}$cells enhance the development of resistance by their ability to produce gamma-interferon (IFN- $\gamma$ ) (Louis) and an optimal balance between the two subsets may be required for maximal protection, as was found by M.A. Mielke (Berlin) for listeriosis and brucellosis.

A different way of stimulating $\mathrm{T}$ cells is used by staphylococcal enterotoxins (SE). The mitogenic T-cell response induced by these and other 'superantigens' is dependent on MHC class II molecules present on APCs but is not MHC-restricted and does not require processing. Different SEs lead to the preferential expansion of $\mathrm{T}$ cells expressing a particular $V_{\beta}$-encoded gene segment of the T-cell receptor (TCR). It has been suggested that the SE, TCR and MHC class II molecules form a trimolecular complex with bivalent SE crosslinking variable parts of the TCR and conserved regions of $\mathrm{MHC}$ molecules (B. Fleischer, Mainz; C. Janeway, New Haven). An alternative model for SE action was proposed by C. Janeway: SE may alter a putative endogenous co-ligand in a way that it crosslinks $V_{\beta}$ of the TCR and $\mathrm{MHC}$ molecules sufficiently to activate a $\mathrm{T}$ cell. The endogenous equivalents of superantigens in mice, the products of the $M l s$ locus, may be a normal constituent of cell interactions.

The complex mechanisms governing processing, presentation and recognition make the question of what characterizes a $T$-cell antigen diffcult to answer. Several studies have suggested that $\mathrm{T}$-cell antigens need not necessarily be proteins. For example, in mice, lipophosphoglycan from L. major induces pro- tection that can be transferred by $\mathrm{CD}^{+}{ }^{+} \mathrm{T}$ cells ( $\mathrm{H}$. Moll, Erlangen) and human $T$ cells bearing the $\gamma \delta$ $T$-cell receptor can respond to mycobacterial components that are resistant to protease digestion ( $\mathrm{K}$. Pfeffer, Munich).

\section{Macrophages as anti-microbial effector cells}

To destroy invading organisms macrophages need to convert from a state that sustains intracellular replication of microbes to an activated state. C. Nathan (New York) summarized recent progress in the understanding of the processes regulating macrophage activation, particularly the biochemical pathways that may be used to generate toxic reactive oxygen and nitrogen intermediates (ROI; RNI). RNI can be regarded as sequential intermediates in the oxidation of a guanidonitrogen from $L$-arginine to nitrate. The microbial target molecules for RNI have yet to be identified but it seems likely that they will be found in metabolic pathways such as electron transport chains, the tricarboxylic acid cycle and DNA synthesis.

The list of cytokines acting on macrophages to induce ROI and RNI and otherwise enhancing anti-microbial activity is long and includes granulocyte-macrophage colony-stimulating factor (GMCSF) and tumour necrosis factor- $\alpha$ (TNF- $\alpha$ ). These powerful mediators must be carefully controlled: elevated and sustained production of TNF- $\alpha$ correlates with the severity of a number of infectious diseases, including shock in bacterial sepsis, although TNF- $\alpha$ seems to have a beneficial role when produced locally and transiently. V. Kindler (Geneva), for example, showed in ex vivo studies that, in mice infected with BCG, TNF $\alpha$ is synthesized locally at the site of inflammation and is essential for granuloma formation and antibacterial macrophage activation. M-L. Lohmann-Matthes (Hannover) reported that TNF- $\alpha$ acts not only in soluble form but also, and perhaps more importantly to local activity, as a membraneassociated molecule.

Dealing with a single cytokine on an isolated population of cells may be a gross oversimplification of events in vivo, as essentially all macrophage-activating cytokines are produced at low concentrations during an ongoing infection. W. Solbach (Erlangen) showed that TNF- $\alpha$ and interleukin 4 (IL-4) in low concentrations independently synergize with IFN- $\gamma$ for macrophage activation whereas none of these cytokines was very effective as a single agent. The cell activation induced by the combination of IL-4 and IFN- $\gamma$ could be blocked completely using antibodies to TNF- $\alpha$ and Solbach, therefore, proposed that IL-4 combined with IFN- $\gamma$ induces the production of TNF- $\alpha$ by macrophages; TNF- $\alpha$ then synergizes with IFN- $\gamma$ to activate the cells for anti-parasite activity.

Since the results of macrophage activation are potentially detrimental to the host, it is not surprising that soluble factors that deactivate macrophages have been discovered. Exciting work from Nathan's group has attributed this activity to macrophage-deactivating factor (MDF) and transforming growth factor $\beta$ (TGF- $\beta$ ). MDF, a $13 \mathrm{kDa}$ protein, has been purified to homogeneity and apparently interferes with $\mathrm{ROI}$ production by reducing the affinity of the enzyme $\mathrm{NADPH}: \mathrm{O}_{2}$ oxidoreductase for the co-substrate NADPH. MDF is distinct from TGF- $\beta$ since neither the antibodies nor the cell surface receptors that bind TGF- $\beta$ recognize MDF.

\section{Vaccination and treatment}

Several speakers discussed the implications of recent findings for vaccination prophylaxis and treatment of infections. A. Sher (Bethesda) recalled that live attenuated vaccines usually induce a better level of protection than purified or recombinant immunogens. This was also testified to by R. Pink (Basel), K. Früh (Heidelberg) and B. Knapp (Marburg), all of whom work on molecularly-defined immunogens from Plasmodium falciparum. Major differences between living and non-living vaccines are antigen persistence in the vaccinee and the inability, so far, to target non-living immunogens to macrophages in a form that induces a protective immune response.

Protective immune responses can be mediated by either $\mathrm{CD}^{+}$or 
$\mathrm{CD} 8+$ cells and in many cases are associated with high levels of IFN- $\gamma$. A powerful vaccine may, therefore, consist of an attenuated living organism that acts as a persisting carrier of defined microbial immunogens from the infectious agent. It should induce an IFN- $\gamma$-biased response and may even incorporate genes for 'beneficial' cytokines or antagonists of 'detrimental' cytokines. Two initiatives of this type were discussed. Sher reported on his success in vaccinating mice with live BCG that contained an integral schistosomal paramyosin gene against schistosomiasis and E. Liew (Beckenham) reported on mice immunized orally with mutant $S$. typhimurium (AroA-) that carried the gp63 gene of $L$. major. This vaccine induced IFN- $\gamma$ production by $\mathrm{CD} 4+\mathrm{T}$ cells and a partial resistance to infection. However, activation of IFN- $\gamma$ mediated responses alone may not always be sufficient to induce protection. J. Langhorne (Freiburg) clearly showed in mouse infections with
Plasmodium chabaudi that, in addition to IFN- $\gamma, \mathrm{CD}^{+}{ }^{+} \mathrm{T}$-celldependent antibody production was essential for complete clearance of the pathogen. Conversely, the presence of high levels of IFN- $\gamma$ does not necessarily indicate protection (Louis). Vaccination against Lyme disease using the outer surface protein A (OspA) of Borrelia burgdorferi induces a protective antibody response in mice (M. Simon, Freiburg) and the same antigen stimulates $T$ cells from patients with Lyme arthritis (G. Burmester, Erlangen). Clearly the type of immune response that gives optimal protection varies with the pathogen For this reason a great deal more information on basic immunesystem-parasite interaction is required before optimized vaccine protocols can be developed.

In addition to active immunization, passive immunoenhancing therapy deserves attention in the future. This approach has proved to be effective in trials of IFN- $\gamma$ and GM-
CSF. G. Kaplan (New York) is now assessing intradermal deposition of IL-2 into lesions of patients with lepromatous leprosy. Another strategy of intervention is the blockade of stimuli inducing detrimental TNF responses, as was successfully performed in patients with Gramnegative sepsis that were given antibodies to lipopolysaccharide (LPS) (B. Baumgartner, Lausanne). A third strategy, that of blocking macrophage deactivation, will be used in the near future.

Clearly vaccine development is an area of great interest and of considerable, if as yet unfulfilled, potential. The increasing awareness of host-parasite interactions will surely supply the momentum for further advancement in the near future.

Werner Solbach, Heidrun Moll and Martin Röllinghoff are at the Institut fïr Klinische Mikrobiologie, Wasserturmstrasse 3, D-8520 Erlangen 3, FRG.

\section{Today's Immunology Today}

In this issue, the first of 1991, we launch a new look for Immunology Today. The aim of the redesign is first and foremost to make the journal more accessible to readers by simplifying the layout, improving the readability of the text and enhancing its appearance. To achieve this we have altered the section names, typeface and logo and introduced a centered page layout and an elegant, clear typeface that is easy on the eye.

One of the most noticeable and appealing changes is to the Contents page, which can now be found on page 3 . The previous 'busy' arrangement has been replaced by a much clearer, more functional design. Section titles have also been altered. Trends, which replaces News and Features, will focus on new directions in immunological research with a combination of short, themed meeting reports and other major news stories. Another purpose of the redesign is to distinguish more clearly between speculative articles and review papers. With this in mind, and to reflect its content more accurately, the Rostrum section has been renamed Viewpoint. In every other respect it is unchanged: it will continue to be the forum for airing new hypotheses, debating controversial topics and exploring the leading edges of immunology. Most of the articles will, as always, be suggested by readers.

Concise, readable and informative reviews commissioned from leading researchers will remain at the heart of each issue. The timeliness and high quality of these articles have helped to make Immunology Today the most popular way for immunologists to stay up to date with this exciting and fast-moving subject.

Editorial pages have been increased to 580 for 1991 and to make the most of this windfall for readers an entirely new section, Kaleidoscope, has been introduced. As the name suggests, Kaleidoscope will contain a rich variety of new articles that will extend the scope of the journal. For example, in this issue the first of an occasional history series, Immunology Yesterday, is featured. Future topics will include articles on national and international immunology organizations, a 'Good Journal Guide', book review supplements, guidelines for science writing and design of figures and insights into the application of immunology in industry. An expanded correspondence section, book reviews and the meetings diary will be regular components of Kaleidoscope.

We would like to extend our thanks and congratulations to Sheila Betts (Graphic Design) who is responsible for the new look and to gratefully acknowledge the input of the Production Department of Elsevier Trends Journals, particularly Judith Bastin, and of Susan Gatell, Marketing Manager.

Comments on the content and suggestions for further improvements to the design will always be welcomed.

Richard B. Gallagher Editor Christine Doberska Assistant Editor 